\title{
ESTUDIO DE LA DIROFILARIOSISCANINA EN LA RIBERA DEL RÍO CHILLÓN, LIMA
}

\author{
Carlos Chipana Q. ${ }^{1}$, Amanda Chávez V. ${ }^{2}$, Eva Casas A. ${ }^{2}$ \\ y Francisco Suárez A. ${ }^{3}$
}

\section{ABSTRACT}

Themain objective of thestudy was to determinethepresenceof dirofilariosis ( $D$. immitis) in districts of Lima located al ong the Chillon river (Puente Piedra, Comas, Carabayllo, Los Olivos and Ventanilla). Blood samples were collected in vacuotainer tubes containing anticoagul ant from 200 dogs of 1-10 years of age that were selected at random, without distinction among breeds and sex. Samples were processed using three diagnostic tests: themethod of microcapillary, Knott modified for microfilarias, and the ELISA test that detects antigens of secretion and excretion of the adult parasite. The overall results indicated $3.2 \pm 2.4 \%$ of infection when using the enzymatic ELISA test, whereas $1.5 \pm 1.7 \%$ when using the Knott modified and the mircocapillary tests. The prevalenceof dirofilariosis in themonitored districts was: $4.4 \%$ in al I threetests in Puente Piedra, 5\% using theELISA testin Ventanilla; 2.5\% inall threetestsin Comas, $2.5 \%$ using the ELISA test en Los Olivos; and $0 \%$ in Carabayllo. It was concluded that dog dirofilariosis does not represent a mayor parasitic problem; however preventive and control measures should not beleft asi dein order to keep the current level under control.

K ey words: dirofilariosis, dogs, D. immitis, ELISA

\section{RESUMEN}

Sedeterminólapresenciadedirofiliarosis ( $D$. immitis) en distritos deLimaribereños al Río Chillón (PuentePiedra, Comas, Carabayllo, LosOlivosy Ventanilla). Setomaron muestras de sangreen vacuotai ners con anticoagulante de 200 perros al azar, sin distinción derazani sexo y con edades comprendi das entre 1-10años. El di agnóstico sereal izó mediantelas pruebas del método del microcapilar, el Knottmodificado paramicrofilarias, y la prueba deELISA que detecta antígenos desecrecióny excreción del parásito adul to. Los resultados mostraron una preval encia en la zona estudiada de $3+2.4 \%$ mediantela pruebaenzimáticaELISA, mientras que por las pruebas demicrocapilar $\mathrm{K}$ Knott modificado seencontró un total de 1.5+1.7\%. Lapreval encia dedirofilariosis en los distritosfue: Puente Piedra con $4.4 \%$ de animales positivos en las tres pruebas; Ventanilla con $5 \%$ donde sólo se detectó presencia de Dirofilaria immitis mediante la prueba de ELISA; Comas con $2.5 \%$ de animales positivos mediante las tres pruebas diagnósticas; LoS

\footnotetext{
${ }^{1}$ Práctica privada

${ }^{2}$ Laboratorio de Microbiología y Parasitología, FMV-UNMSM. E-mail: a chavez g@hotmail.com

${ }^{3}$ Laboratorio de Medicina Preventiva, FMV-UNMSM
} 
Olivos $2.5 \%$ de perros positivos mediantela prueba de ELISA; y Carabayllo con $0 \%$. Se concluye que la dirofilariosis canina en la zona de estudio no constituye un grave problema parasitario; sin embargo no debe descuidarse las medi das de prevención y control a fin de evitar su aumento y propagación.

Palabras clave: dirofilariosis, perros, D. immitis, ELISA

\section{INTRODUCCIÓN}

La dirofilariosis canina o enfermedad del gusano del corazón, es ocasionada por el nemátodo Dirofilaria immitis; cuya forma adulta se localiza en el lado derecho del corazón y arterias pulmonares desus hospederos definitivos, como el perro y el gato. Esta enfermedades dedistribución mundial, decurso general mentecrónico y subclínico, lo que influye que haya pacientes que no reciban tratamiento oportuno, o que lo reciban sólo cuando presentan signos clínicos que hacen sospechar dedi rofilariosis (Kirk y Bonagura 1996; Schrey y Trautvetter, 1998).

La dirofilariosis es transmitida por mosquitos vectores perteneciente a los génerosAedes, Anopheles, Cul exy Taeniorhynchus; pudiendo eventualmenteafectar al hombre (enfermedad zoonótica), el que actúa como un hospedero accidental (Aiello, 1998; Leguía 1996).

Existen antecedentes dedi rofilariosis en Perú, así Acha (1952) reportó una prevalencia del $6 \%$ en 100 perros sacrificados en laciudad de Lima, y Hernández (1958) informó una prevalencia de $8.8 \%$ en 204 muestras de sangre de perros en Lima, en tanto queen unestudio realizado por Bellido (1995) en criaderos de perros en Lima, encontró una prevalencia de $2 \%$ en 147 muestras de sangre analizadas. En estos estudios, Ios diagnósticos se realizaron con técni cas de observación di recta demicrofilarias, lascuales pre sentan limitaciones porque dependen delahabilidad técnica y de un horario especial para la toma de muestra. En el presente estudio, además delas pruebas convencionales deobservación directa como las de microcapilar (o de Woo) y Knott modificado, se incluye la prueba de diagnóstico serológico de ELISA, pruebadealtasensibilidad yespecificidad(Kirk y Bonagura, 1996), que detecta antígenos de secreción y excreción delas formas adultas de Dirofilaria immitis. La prueba de ELISA permite procesar grandes cantidades de muestras al mismotiempoy no requieredemayor habilidad técnica o de un horario restringido para la toma de muestra.

El objetivo del presente trabajo fue evaluar la presencia de Dirofilaria immitis enlos distritos ribereñosal Río Chillón.

\section{ATERIALES Y MÉTOdOS}

Localidad y toma de muestras

El estudio serealizó en los distritos de la ribera del río Chillón (Ventanilla, Puente Piedra, Comas, Carabaylloy Los Olivos), de la ciudad de Lima.

Se colectaron muestras de sangre de 200 perros, sin distinción de sexo o raza y cuyas edades variaban desde uno hasta 10 años. Seanotóinformación sobrelapermanenciadel animal dentro delacasa del propietario.

La colecta de sangre se realizó entre entre las 16:00 y las 18:00 horas y durantelos meses deenero ajulio de año 2000. El punto deextracciónfuelavenacefálicaantebraquial utilizando vacutainersconanticoagulante.

La muestras fueron procesadas en el Laboratorio de Parasitología de la Facultad de Medicina Veterinaria de la UNMSM. Se 
utilizaron las pruebas diagnósticas para Dirofilaria immitisy sumicrofilaria, lascuales fueronELISA, Knottmodificada, y microcapilar.

El método de microcapilar (Woo)

Para observar microfilarias vivas se realizó un examen de hematocrito convencional, utilizando microcapilares, donde las microfilariasseobservaron principal menteen la capaflogística. La muestras positivas fue ron sometidas a un examen morfológico y biométrico para su diferenciación con la microfilaria de Dipetalonema reconditum, otra filaria común en nuestro medio, no patógena, cuyas formas adul tas se alojan en el subcutáneo del hospedero.

Desarrollo del método de K nott modificado

Se observó las microfilarias muertas, en una submuestra de $1 \mathrm{ml}$ de sangre entera donde se añadió una solución de formol al 2\%. La muestra secentrifugó y el sedimento seobservó al microscopio. En los casos positivos se recurrió a la medición y diferenciación entre la microfilaria de Dirofilaria immitis y la de Dipetalonema reconditum

Desarrollo de la prueba de ELISA

Se utilizó un kit comercial de ELISA (Dirochek ${ }^{\circledR}$ ), el cual detecta antígenos dese creción y excreción de Dirofilaria immitis (especificidad de $100 \%$ y sensi bi lidad de 85.7 a 100\%).

\section{RESULTADOS Y DISCUSIÓN}

El porcentajedeseropositivos mediantela prueba deELISA fuede $3.0+2.4$ mientras que con las pruebas de microcapilar y Knott modificado se detectó una positividad del $1.5+1.7 \%$ (Cuadro 1 ).

La preval encia encontrada en este estudio es relativamente baja comparada con estudios anteriormente real izados bajo otras condiciones (Acha, 1952; Hernández, 1958) o casi iguales (Bellido, 1995); en ésteúltimo caso en canes de criaderos. En todo caso, se debe tomar en cuenta que en estos estudios seutilizaron úni camente pruebas de diagnóstico de observación directa de microfilarias de Dirofilaria immitis.

La diferencia de los porcentajes de diagnóstico entre ELISA y los métodos del microcapilar y K nott modificado pueden deberse a la presentación de infecciones ocultas, donde no se observan microfilarias en sangre. Esto puede deberse a un tratamiento microfilaricida, reacciones dehipersensibilidad contra las microfilarias, infecciones unisexuales, vermes adultos estériles o vermes en local izaciones ectópicas que sólo

Cuadro 1. Presencia de la microfilaria de Dirofilaria immitis (en porcentaje de positivos) según las pruebas de Microcapilar, Knott Modificado y ELISA, en los distritos de la ribera del río Chillón, Lima (enero-julio del 2000)

\begin{tabular}{lcccc}
\hline \multirow{2}{*}{ Distritos } & \multirow{2}{*}{ Total dePerros } & \multicolumn{3}{c}{ Técnica } \\
\cline { 3 - 5 } & & Microcapilar & Knott Modificado & ELISA \\
\hline PuentePiedra & 45 & 4.4 & 4.4 & 4.4 \\
Comas & 40 & 2.5 & 2.5 & 2.5 \\
Carabayllo & 35 & 0 & 0 & 0 \\
Los Olivos & 40 & 0 & 0 & 2.5 \\
Ventanilla & 40 & 0 & 0 & 5 \\
\hline Total & 200 & $1.5 \pm 1.7$ & $1.5 \pm 1.7$ & $3.0 \pm 2.4$ \\
\hline
\end{tabular}


Cuadro 2. Presencia de Dirofilaria immitis según el tiempo de permanencia de los perros en el hogar, en los distritos ribereños al río Chillón (Puente Piedra, Comas, Carabayllo, Los Olivos y Ventanilla), Lima, enero-julio 2000

\begin{tabular}{lccc}
\hline \multicolumn{1}{c}{$\begin{array}{c}\text { Permanencia en } \\
\text { d hogar }\end{array}$} & $\begin{array}{c}\text { Total de } \\
\text { Perros }\end{array}$ & $\mathrm{n}$ & $\%$ \\
\hline Todo ed día & 14 & 0 & 0 \\
Algunas horas & 186 & 6 & 3.2 \\
\hline Total & 200 & 6 & 3.0 \\
\hline
\end{tabular}

habrían sido detectadas por la prueba de ELISA. Caberesaltar que la muestra desangre para realizar la técnica de ELISA que detecta antígenos de secreción y excreción de Dirofilaria immitis, puede ser recolectada en cual quier momento del día, a diferencia de las pruebas de microcapilar y Knott modificado que tienen como principal desventaja la restricción de horario para la toma de sangre (6 a 8 pm). Además, se requiere real izar un diagnóstico diferencial de lasmicrofilarias, yaqueenel pásexistelafilaria no patógena Dipetalonema reconditum

Puente Piedra fue uno de los distritos donde se detectó el mayor número de casos positivos. Esto sedebió probabl ementea que en los lugares cercanos a la ribera del Río Chillónexistenmuchos cultivos depan llevar, y debido al continuo regadío ocurren empozamientos de agua o pequeñas desvia ciones del cauce del río, quefavorecelaconcentración demosquitos vectores aumentando la probabilidad decontagio. Ventanillaes el otro distrito que presenta un el evado porcentaje de perros positivos (solamentecon la prueba de ELISA) debido probablemente a la fal ta de un adecuado sistema de desagüe que ocasiona empozamientos de agua, ele vándosetambién la probabilidad de contagio por medio delos mosquitos.

Los seis perros positivos del grupo de animales que permanecían fuera del hogar las 24 horas eran perros callejeros (Cuadro 2), y por lo tanto, expuestos al contagio por
Ios mosquitos vectores de esta enfermedad, ya que estos perros se al imentaban de los desperdicios que se arrojaban a las riberas del río Chillón por los pobladores delaszonas al edañas.

\section{CONCLUSIONES}

Los porcentajes de reactores halla dos mediante la prueba de ELISA fueron de $3 \%+2.4$ IC y de $1.5 \%+1.7$ IC con las técni cas demicrocapilar y K nottmodificado. Estosval ores seconsideran bajos, lo quepermiteconcluir quela di rofilariosiscaninaenla zona de estudio no constituye un grave problema sanitario. Sin embargo, no debe descuidarsesu monitoreo y control a fin deevitar su aumento y propagación.

\section{L iteratura Citada}

1. Aiello, S. 1998. The Merck veterinary manual. 8a ed. p. 93-97. Ed. Merck \& Co., Inc. USA.

2. Acha, P. 1952. Porcentajedeparasitosis del Canis familiaris en la ciudad de Lima Tesis Bach., Fac. Med. Vet, Univ. Nac. Mayor deSan Marcos, Lima 35 p.

3. Bellido, M.E. 1995. Prevalencia de Dirofilaria immitis en criaderos de pe ros (Canis familiaris) en Lima Metropolitana Tesis Bach., Fac. MedVet, Univ. Nac. Mayor deSan Marcos, Lima. 38p. 


\section{Chipana etal.}

4. Hernández, A. 1958. Contribución al estudio delafilariasiscaninaenlaciudad de Lima. Tesis Bach., Fac. Med. Vet., Univ. Nac. Mayor deSan Marcos, Lima $29 \mathrm{p}$.

5. Kirk, R.; J . Bonagura. 1996. Terapéur tica veterinaria de pequeños animales. $11^{a}$ ed. p 903. Ed. Intermédica, España.
6. Leguía, G. 1996. Enfermedades parasitarias deperros y gatos. Epidemiología y control. $1^{\text {ra }}$ ed. p 128. Editora del Mar. Perú.

7. Schrey, C.F.; E. Trautvetter. 1998. Dirofilariosiscaninay felina- Diagnósticoy tratamiento. Whal tamFocus 8: 2330. 